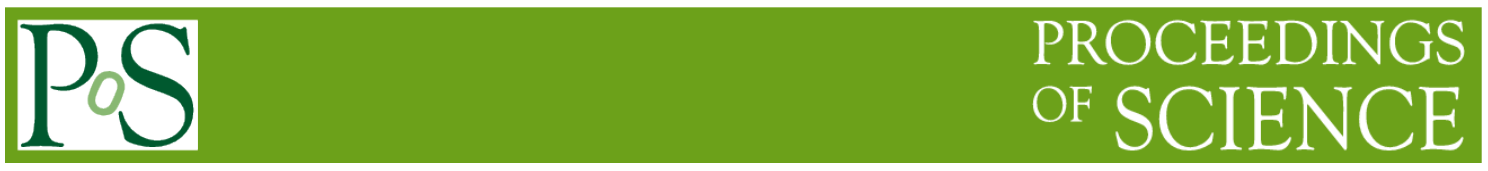

\title{
Pressure Sensing Insoles for Learning Tai-Chi Chuan
}

\section{Yu-Chun Chen ${ }^{1}$}

National Taiwan University, Taipei, 10617, Taiwan

E-mail: r01944022@csie.ntu.edu.tw

\section{Peng-Yuan Kao}

National Taiwan University, Taipei, 10617, Taiwan

E-mail: zbabqrahotmail.com

\section{Kuan-Yin Lu}

National Taiwan University, Taipei, 10617, Taiwan

E-mail: r03922088@csie.ntu.edu.tw

\section{Shih-Yao Wei}

Institute for Information Industry, Taipei, 105, Taiwan

E-mail: syweidiii.org.tw

National Taiwan University, Taipei, 10617, Taiwan

E-mail: d04944010@csie.ntu.edu.tw

\section{Yi-Ping Hung ${ }^{2}$}

National Taiwan University, Taipei, 10617, Taiwan

E-mail:_hung@csie.ntu.edu.tw

In this work, we designed and implemented a pair of pressure sensing insoles to quantize the user's plantar pressure distributions. Each pressure sensing insole includes four force sensor modules, a microcontroller and a wireless data transmission module. We firstly performed a simple process to calibrate the force sensor, and then built a plantar pressure model for estimating the complete plantar pressure distribution. We have applied the pressure sensing insoles to develop a Tai-Chi Chuan instruction system which, with visual feedback, can help users to learn the correct plantar pressure distribution of the fundamental movements of Tai-Chi Chuan. We have also evaluated the efficiency of learning Tai-Chi Chuan with our system. According to our experimental results, users using our system can learn faster than those without using our system.

ISCC 2015

18-19, December, 2015

Guangzhou, China

${ }^{1}$ This work is partially supported by the III Innovative and Prospective Technologies Project of the Institute for Information Industry which is subsidized by the Ministry of Economy Affairs of Taiwan, and by the Ministry of Science and Technology, Taiwan, under Grants MOST 104-2627-E-002-001.

${ }^{2}$ Speaker 


\section{Introduction}

Tai Chi Chuan (or TCC for short) is a famous ancient Chinese martial art which can benefit people; however, for many people who have tried to learn TCC, it turns out to be not so easy as they thought at the beginning. The traditional ways to learn TCC are reading TCC manuals, watching demonstration videos, or physically taught by a coach. Due to the complexity of TCC learning, students often spend a long time in observing and comprehending the correct plantar pressure distribution. To help people learn the correct plantar pressure distribution of fundamental TCC movements, we have designed and implemented a pair of pressure sensing insoles to measure quantitatively the plantar pressure distributions of the students and the coach. With our TCC instruction system, students can compare their plantar pressure distributions with those of the coach while practicing TCC.

Our main contribution in this paper is to design and implement a pair of plantar pressure insoles and then apply it to a TCC instruction system. Section 2 presents a literature review of pressure sensing insoles and TCC instruction system. Section 3 provides the implementation details of the pressure sensing insoles, and Section 4 introduces our TCC instruction system. The experimental results shown in Section 5 demonstrates that the students using our system can learn TCC faster than those without our system. Section 6 gives the conclusion and suggests some of the future work.

\section{Related Work}

Pressure sensing insoles have been developed for decades. Many in-shoe plantar pressure measurements are implemented for different applications such as the gait phase detection [1][2] [3]. or medical treatment [4]. Morriset et al. [5] developed a real-time feedback system for gait analysis and their shoes consist of many kinds of sensors like force sensitive resistor and band sensor to analyze gait information. Some applications are for Dance instruction like Paradiso [6]. The dance instruction shoes contain not only FSR and the bend sensor but also 3-axis shock accelerometer for compleicate dance movements. There have been some related products on the market such as Nike+ sensor and Nike+ Pressure Sensor. Nike+ sensor by using a piezoelectric sensor is a pedometer, and Nike+ Pressure Sensor contains four embedded pressure sensors and tri-axial accelerometer for gait analysis like jump.

Because it is not so easy to learn TCC, some researchers have tried developing TCC instruction systems to help students to learn TCC. For example, Chua et al. [7] built a full-body training system in virtual reality environment and created an avatar of the coach in front of students. Students could watch the coach's movements and compare their own movements to the coach's. Moreover, Jin et al.[8] proposed their Kinect-based system which can help the students improve their performance on learning TCC. The Kinect-based system evaluates the students' performance automatically and provides feedback for correction in real-time by measuring the difference between the coach's posture and the student's.

Also, the design of visual feedback is also important for our purpose. Femery et al. [9] implemented a system to give visual and audio feedback to alarm users and correct their plantar pressure distribution patterns. Yu et al. [10] developed a multimedia-assisted system including the visual feedback and delivering the walking and breathing information on the smartphone to 
help users learn walking meditation. In their experiment, the results show that visual-auditory mechanism is better to help beginners decrease incorrect footsteps, the stepping retardation time, and the breathing retardation time; besides, it can also enhance the walking stability during the walking meditation. Recently, Nylander et al. [11] implemented RunRight system which created a visualization of the running movement based on acceleration in vertical and horizontal direction. In their experiments on user experience, the six subjects all found it easy to understand the visual feedback.

\section{Pressure Sensing Insoles}

The pressure sensing insole we developed includes four force sensor modules, a microcontroller and a wireless data transmission module. The microcontroller collects the plantar pressure data from the force sensor modules and downscale every pressure data from 1024 to 256 (one byte) to reduce the loading of data transfer. After data processing, the transmission module sends the processed data via a Bluetooth module. All data are transmitted at the $100 \mathrm{~Hz}$ frequency. The hardware structure is shown in Fig. 1(a).

Each force sensor module is comprised of high-and-low resolution force sensors. High and low resolution force sensors have different force dynamic ranges. These two kinds of sensors are designed for two different ranges of pressure sensing. When we use our foot to touch the floor lightly, we need the high resolution sensor for sensing the small pressure variation; on the other hand, when we use our foot to step on the floor heavily, we need the sensor for sensing large pressure variation, which can only provide lower resolution. The high and low resolution force sensors in each force sensor module are mounted at the same place. As shown in Fig.1(b), the four force sensor modules are mounted into the insole at the heel, the first metatarsal head, the fifth metatarsal head and the great toe. The prototype of pressure sensing insoles is shown in Fig.1(c).

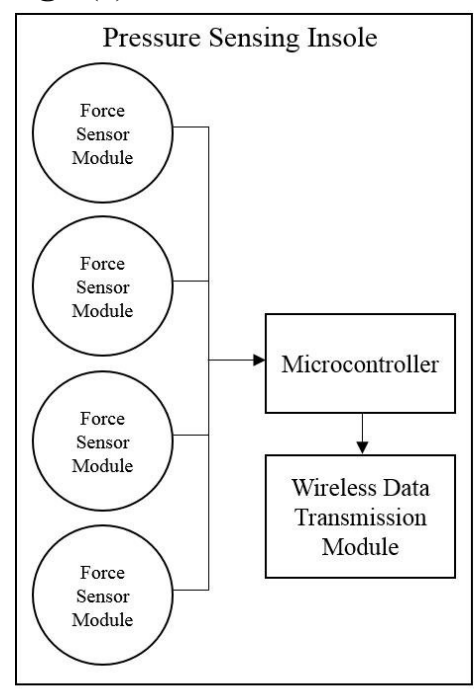

(a)

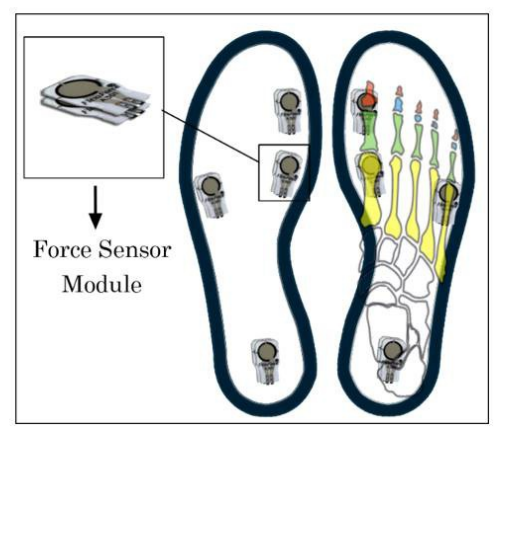

(b)

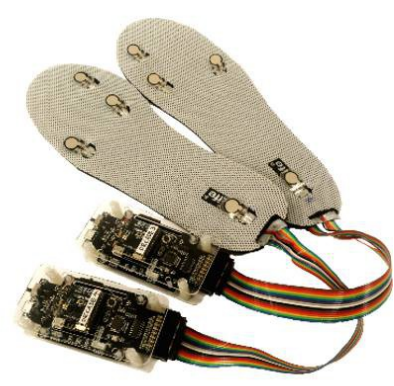

(c)

Figure 1: Pressure Sensing Insoles: (a) the hardware structure; (b) the placement of force sensors. (c) the prototype of pressure sensing insoles. 


\subsection{Force Sensor Calibration}

First, a slug smaller than the sensing area of the force sensor is put on the force sensor, and then we put them on a platform scale and apply a known load on them. At the same time, we record the output of the platform scale and the force sensor value. Then we repeat the above steps and get many outputs of the platform scale and corresponding force sensor values. Then we can use the above data to fit a curve which is:

$$
f(x)=a \cdot e^{(b \cdot x)}+c \cdot e^{(b \cdot x)}
$$

where $a=0.4768 e^{-10}, b=0.1006, c=0.05773, d=0.01462$ for the fitting curve of high resolution force sensor, and $a=6.779 e^{-10}, b=0.1176, c=0.1401, d=0.0144$ for the fitting curve of low resolution force sensor.

The result of the calibration is shown in Fig.2, which can be stored as a look-up table which can be used to look up the correspondence between the force sensor values and the forces. While the loading is greater than about two kilograms, we look up the table of the low resolution force sensor, or we look up high resolution force sensor's look-up table.

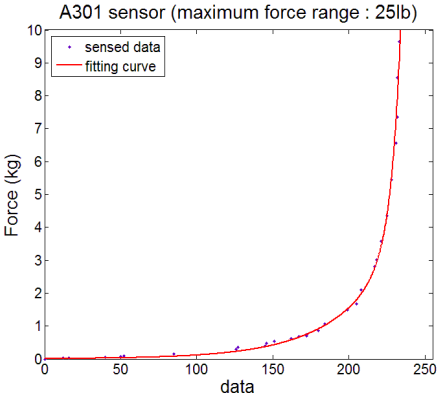

(a)

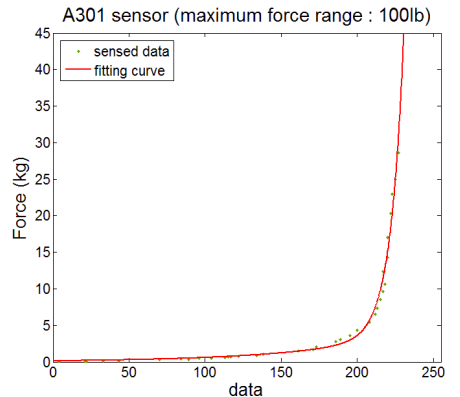

(b)

Figure 2: Correspondence of Force Sensor Values (data in the figure) and forces. (a) high resolution sensor. (b) low resolution sensor. Notice that the scaling of the vertical axes are different.

\subsection{Plantar Pressure Model}

Our task is to estimate the complete plantar pressure distribution based on the measurement acquired from the four positions where the force sensors are embedded, because we only take measurement of the pressures on the four positions. What we did was as follows.

We first got footprints of two subjects. According to the positions we put the force sensor modules, we divided the footprints into four parts as shown in Fig.3(a). We found that the bottom areas of four parts of footprints between two subjects were similar, so we made four wood of bottom areas of four parts as shown in Fig.3(b). Then we repeated the calibration described in Section 3.1. The result of calibration is shown in Fig.3(c) and Fig.3(d). We can find the raw data of three parts except toe are mixing, thus we assumed that their proportion of total plantar pressure were the same and we fit two curves for the toe and other part. Based on the result of the fitting curves, we can also create a look-up table and use it while receiving the pressure data from the insoles. 


\subsection{Zero Correction}

Users have to do the zero correction because it may exist pressure between their feet and pressure sensing insoles while wearing them. Users only need to vacate their feet first and let our system record the initial value of each sensor to do the zero correction.

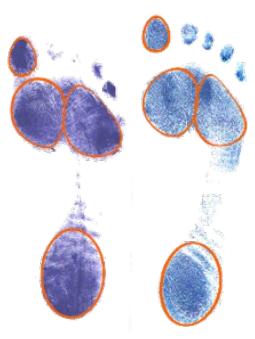

(a)

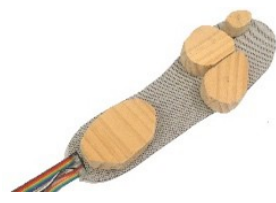

(b)

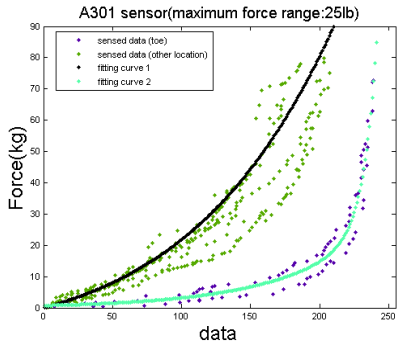

(c)

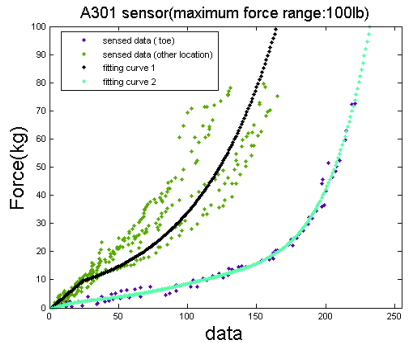

(d)

Figure 3: Plantar Pressure Model: (a) the footprints of two subjects. (b) the corresponding wood of the footprints of two subjects. (c)(d) the correspondence of high and low resolution force sensor values (data) and forces in the Plantar Pressure Model.

\section{TCC Instruction System}

In this section, we introduce our TCC instruction system. In the TCC, the plantar pressure distribution is one of the most important issues, but it is too difficult to realize how to achieve the standard plantar pressure distribution (i.e. TCC coach's plantar pressure distribution) by only observing coach's movement or listening to coach's description; therefore, we let the students wear pressure the sensing insoles and watch the visual feedback to learn how to achieve the standard plantar pressure distribution of TCC's fundamental movements.

After wearing the pressure sensing insoles and let the insoles connect to the tablet, students should do the zero correction. Then students can watch the differences of their and coach's plantar pressure distributions. The operating process of TCC instruction is shown in Fig.4.

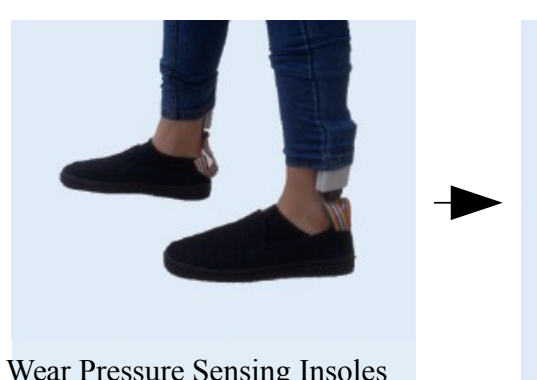

Wear Pressure Sensing Insoles
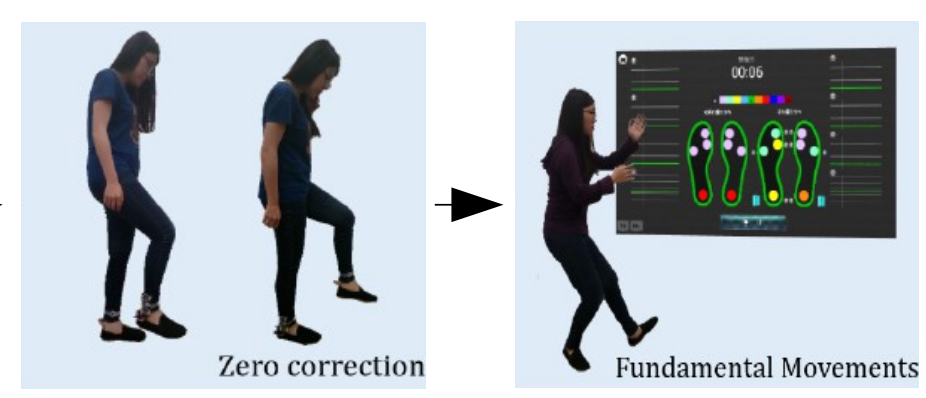

Figure 4: Operating Process of Our TCC Instruction System.

\subsection{Visual Feedback}

The visual feedback of the pressure sensing insoles is shown in Fig. 5. The plantar pressure is divided into 10 levels. Each level represents the proportion of the total pressure of a foot. We use 10 different colors to represent these 10 levels. 
There are four circles on a foot. Each circle's color represents the proportion of the total pressure of the foot at that position. The color of the foot's contour represents the proportion of the total pressure of two feet. The curves on the left side and right side of Fig. 5. is used to show the stability of the pressure of eight positions of two feet.

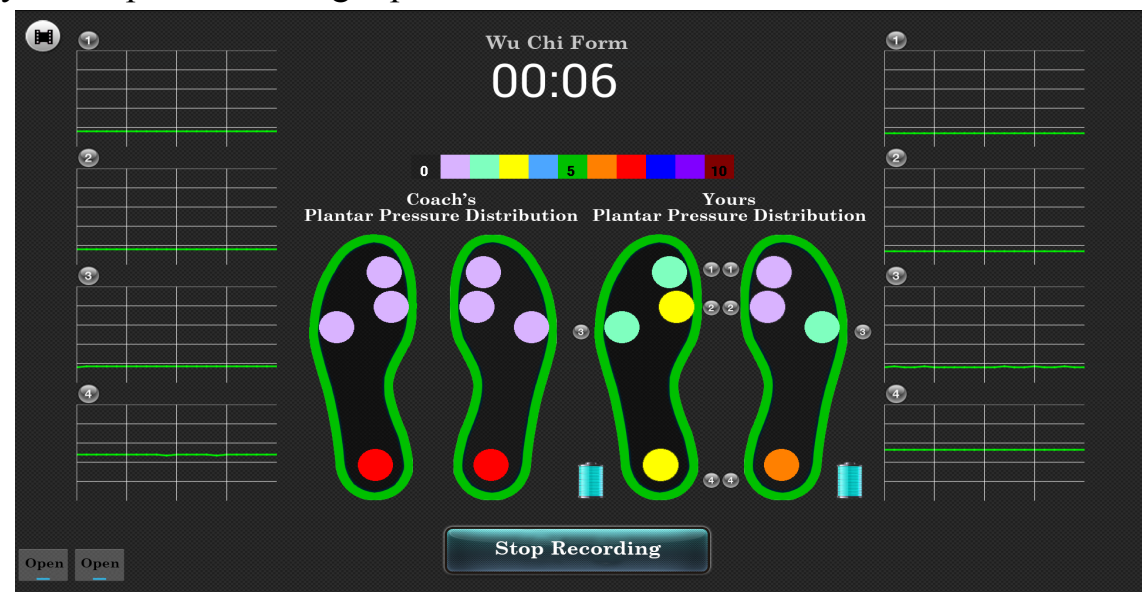

Figure 5: Visual Feedback of the Pressure Sensing Insoles.

\subsection{Pressure Evaluation}

The plantar pressure distribution of the student is compared with the standard plantar pressure distribution by the following method. We calculate the distance of plantar pressure distribution between students' and the standard in the eight-dimensional space. Each dimensional represents the information of an insole sensor module. We normalize the input observation sequence by the body weight and filter the data by high-pass filter in frequency domain and then transform back to the time domain. After normalization, each data represents the proportion of user's body weight in a part of the foot. We suppose that the input sequence processed is represented by $S(i)$ where $i=1, \ldots, n$ and a reference vector of the standard plantar pressure distribution $T$. Students can adjust the practice time flexibly, so $n$ is not constant. The distance between the student's and the standard $D(i)$ at sampling instant ${ }_{i}$ can be calculated by:

$$
D(i)=\sqrt{\sum_{k=1}^{8}(S(i, k)-T(k))^{2}},
$$

where $S(i, k)$ is the ${ }_{k}$-dimensional information of student at sampling instant ${ }_{i}$; therefore, the average distance of this integral practice time Diff can be calculated by:

$$
\text { Dif } f=\frac{\sum_{i=1}^{n} D(i)}{n}
$$




\section{Experiments}

We invited six subjects to learn TCC with pressure sensing insoles in the shoes. There were three of six subjects who have learned TCC before. We divided these six subjects into the experiment and the control group. The information of these subjects was shown in Table 1. In this experiment, we analyzed how fast they can do "Hold the Lute", a movement of TCC, to evaluate the efficiency.

Before starting the experiment, we taught all subjects how to do the movement and what was the right plantar pressure distribution of the movement in the traditional teaching way for three minutes.

\begin{tabular}{|c|c|c|c|c|c|c|}
\hline & \multicolumn{3}{|c|}{ Experiment Group } & \multicolumn{3}{c|}{ Control Group } \\
\hline Subjects & Subject 1 & Subject 2 & Subject 3 & Subject 4 & Subject 5 & Subject 6 \\
\hline $\begin{array}{c}\text { Learned TCC } \\
\text { Before (Y/N) }\end{array}$ & $\mathrm{N}$ & $\mathrm{N}$ & $\mathrm{Y}$ & $\mathrm{N}$ & $\mathrm{Y}$ & $\mathrm{Y}$ \\
\hline
\end{tabular}

Table 1: Information of the Subjects.

After resting about ten minutes, all subjects did the same movement as fast as possible for six times with pressure sensing insoles in the shoes. Every subject had to achieve and maintain the movement for three seconds. The subjects in the experiment group did the movement with visual feedback, but the subjects in the control group did the movement without visual feedback.

We analyzed the experimental data by the method described in Section 4.2. If the distance $D(i)$ at a sampling instant was less than a threshold we determined, we said that the subject achieved the standard plantar pressure distribution. The time of achieving the standard plantar pressure distribution should be maintained for 3 seconds and then we said that the subject completed the movement successfully.

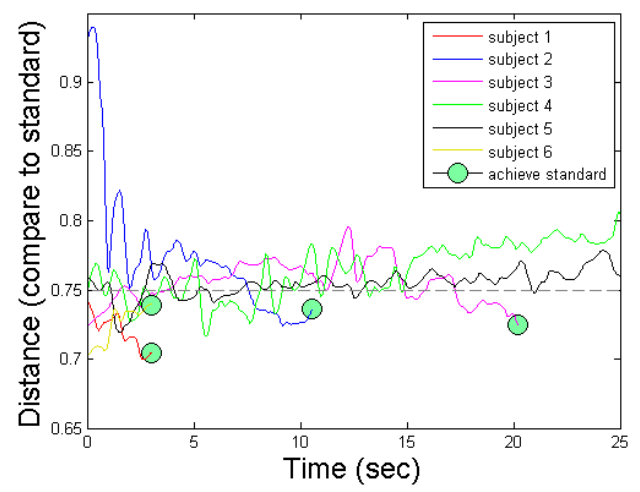

Figure 6: This figure shows the distance of the plantar pressure distribution between every subject and the standard when the subject performed the TCC movement. The green ball indicates the time instant that the subject achieved the standard pressure distribution.

From Fig. 6, we can find that all subjects in the experiment group completed successfully and spent less time than those in the control group. Only Subject 6 in the control group achieved the standard plantar pressure distribution successfully. On the average, the users who had used the pressure sensing shoes with visual feedback to learn TCC have spent less time than those without visual feedback. We shall perform more experiments in the future. 


\section{Conclusions \& Future Works}

In this paper, we have introduced a pair of pressure sensing insoles for sensing the foot plantar pressure distribution, and also applied it to develop a TCC instruction system to help people to learn TCC. We have demonstrated that, for subjects using our system to learn TCC, their improvement is better than those without the visual feedback on average.

Currently, we are working on enhancing our TCC instruction system with head-mounted display, so that the information obtained by the pressure sensing insoles can be displayed on the head-mounted display, and hence, it can be seen easily by the users when they rotate their body while practicing TCC.

\section{References}

[1] K.youngchul Kong and M. Tomizuka. Smooth and continuous human gait phase detection based on foot pressure patterns. IEEE International Conference on Robotics and Automation, Pasadena, CA. 3678-3683. (2008)

[2] M. Chen, B.F. Huang, and Yangsheng Xu. Intelligent shoes for abnormal gait detection. IEEE International Conference on Robotics and Automation, Pasadena, CA. 2019-2024. (2008)

[3] D. Novak, P. Reberšek, S. Marco, M. DeRossi, M. Donati, J. Podobnik, T. Beravs, T. Lenzi, N. Vitiello, M. C. Carrozza, and M. Munih. Automated detection of gait initiation and termination using wearable sensors. Medical engineering \& physics, 35(12):1713-1720. (2013)

[4] M.W. Lawless, G. T. Reveal, and R. T. Laughlin. Foot pressures during gait: a com-parison of techniques for reducing pressure points. Foot\&ankle international, 22(7):594-597.(2001)

[5] Stacy J Morris and Joseph A Paradiso. Shoe-integrated sensor system for wireless gait analysis and real-time feedback. 24th Annual Conference and the Annual Fall Meeting of the Biomedical Engineering Society, EMBS/BMES Conference, Houston, TX, USA, 3:2468-2469.(2002)

[6] J. A .Paradiso. Footnotes: personal reflections on the development of instrumented dance shoes and their musical applications. Quinz, E(ed) Digital Performance, Anomalie, 2:34-49. (2002)

[7] Philo Tan Chua, Rebecca Crivella, Bo Daly, Ning Hu, Russ Schaaf, David Ventura, Todd Camill, Jessica Hodgins, and Randy Pausch. Training for physical tasks in virtual environments: Tai chi. In Proceedings IEEE Virtual Reality, 87-94. (2003)

[8] Y. Jin, X.X. Hu, and G.S. Wu. A Tai Chi Training System Based on Fast Skeleton Matching Algorithm. In Computer Vision-ECCV 2012. Workshops and Demonstrations. Springer, Berlin, Heidelberg. 667-670. (2012).

[9] V. G. Femery, P. G. Moretto, J.-M. G. Hespel, André Thévenon, and Ghislaine Lensel. A real-time plantar pressure feedback device for foot unloading. Archives of physical medicine and rehabilitation, 85(10):1724-1728. (2004).

[10] M.-C. Yu, H. Wu, M.-S. Lee, and Y.-P.g Hung. Multimedia Assisted Breathwalk-aware System. IEEE Transactions on Biomedical Engineering, 59(12): 3276-3282,Oct.(2012)

[11] S. Nylander, M. Jacobsson, and J. Tholander. Runright: real-time visual and audio feedback on running. In ACM CHI'14 Extended Abstracts on Human Factors in Computing Systems. 583-586. (2014) 